\title{
INTERPRETATIONS OF RADIO AND OPTICAL OBSERVATIONS OF NGC 1275*
}

\author{
J. H. OORT \\ Sterrewacht, Leiden, The Netherlands
}

\begin{abstract}
An essential part of the following report is based on new spectrograms taken by Vera C. Rubin, W. Kent Ford, and Charles J. Peterson with the 4-meter Kitt Peak telescope and its RC image-tube spectrograph. These data, which they have kindly made available for the present occasion, will be discussed in detail in a forthcoming article.
\end{abstract}

The principal component of NGC 1275 is a giant E galaxy. It is surrounded by emission filaments extending in all directions to roughly $50 \mathrm{kpc}$. As was first shown by Minkowski (1955) the inner filaments within about $20 \mathrm{kpc}$ from the center - have velocities concentrated around two widely separated values, one around $+5200 \mathrm{~km} \mathrm{~s}^{-1}$, which is the velocity of the $\mathrm{E}$ galaxy, the other around $+8200 \mathrm{~km} \mathrm{~s}^{-1}$. Within either group the radial-velocity dispersion is about $\pm 100 \mathrm{~km} \mathrm{~s}^{-1}$. The distribution on the sky is different for the two groups: while the filaments at $5200 \mathrm{~km} \mathrm{~s}^{-1}$ show a more or less circular symmetry around the center of the E galaxy, all $+8200 \mathrm{~km} \mathrm{~s}^{-1}$ filaments lie to the north of this center in a roughly elliptical region of about $50^{\prime \prime} \times 15^{\prime \prime}$ (or $25 \times 8 \mathrm{kpc}$, if a distance of $100 \mathrm{Mpc}$ is assumed) (cf. E. M. and G. R. Burbidge 1965; and C. R. Lynds 1970).

There can be little doubt that the $+5200 \mathrm{~km} \mathrm{~s}^{-1}$ filaments have been expelled from the nucleus of the $\mathrm{E}$ galaxy. The main expulsion probably occurred about 100 million years ago, but it might have continued over a long period. The nucleus is still, or again, active at present; it is a bright radio source, in which VLBI observations have shown an intricate fine-structure, aligned to the north-south direction and extending over about $10 \mathrm{pc}$. There is also a radio halo, of which at least the inner parts show a strong elongation in the same direction. NGC 1275 is an intrinsically strong X-ray source. It is likewise a strong infrared source.

Three different interpretations have been suggested to explain the existence of the two velocities in the emission features.

A. There are two galaxies, an $\mathrm{E}$ or early-type spiral and a late-type galaxy, with a relative velocity of $3000 \mathrm{~km} \mathrm{~s}^{-1}$ in collision with each other (Minkowski 1957).

B. The $8200 \mathrm{~km} \mathrm{~s}^{-1}$ features belong to a non-

*A summary of one of the invited papers presented at the Rudolph Minkowski Symposium, A.S.P. Summer Meeting, Berkeley, 18-22 May 1976. colliding, accidentally superimposed galaxy.

C. The $8200 \mathrm{~km} \mathrm{~s}^{-1}$ knots have been ejected, like the $5200 \mathrm{~km} \mathrm{~s}^{-1}$ filaments (E. M. and G. R. Burbidge 1965).

In $(\mathrm{C})$ the $8200 \mathrm{~km} \mathrm{~s}^{-1}$ features must evidently lie behind the $\mathrm{E}$ galaxy, and even at a considerable distance because otherwise one would expect to see a systematic change in velocity from one side to the other. The fact that this change is no more than 200 $\mathrm{km} \mathrm{s}^{-1}$ indicates that their distance from the ejection point must be more than about $40 \mathrm{kpc}$.

NGC 1275 is full of absorption patches (cf. Minkowski 1968, Plate S-III, right). These lie evidently in front of the $\mathrm{E}$ galaxy. If it can be shown that the absorption features and the $8200 \mathrm{~km} \mathrm{~s}^{-1}$ filaments are connected the latter must likewise lie in front, and cannot be expelled, because in that case they would have to lie at least $40 \mathrm{kpc}$ behind it. There is indeed strong evidence that the absorption patches and the $8200 \mathrm{~km} \mathrm{~s}^{-1}$ filaments belong together. Both occur exclusively in the northern half of the nebula and are indeed confined to very much the same elongated region. Further evidence for a connection between the two is furnished by Rubin, Ford, and Peterson's (RFP) spectrograms. A rather convincing example may be seen on a spectrogram with the slit approximately east-west and passing about $12^{\prime \prime}$ north of the nucleus of the E galaxy (Fig. 1). Near the maximum of the continuum intensity there is a pronounced dust streak absorbing both the main galaxy's continuum and its emission lines. A striking emission feature of the $8200 \mathrm{~km} \mathrm{~s}^{-1}$ system is observed at practically the same position, which strongly indicates a connection between the two. There is generally a correlation in position between the absorbing knots and the highvelocity emission. In the region around the nucleus of the E galaxy the connection between absorption and $8200 \mathrm{~km} \mathrm{~s}^{-1}$ emission is indicated in a somewhat different manner. As had previously been observed by Minkowski, the core of the $\mathrm{E}$ galaxy has a prominent A-type spectrum. Our spectrogram through the nucleus (Fig. 2) shows the A-type features quite 

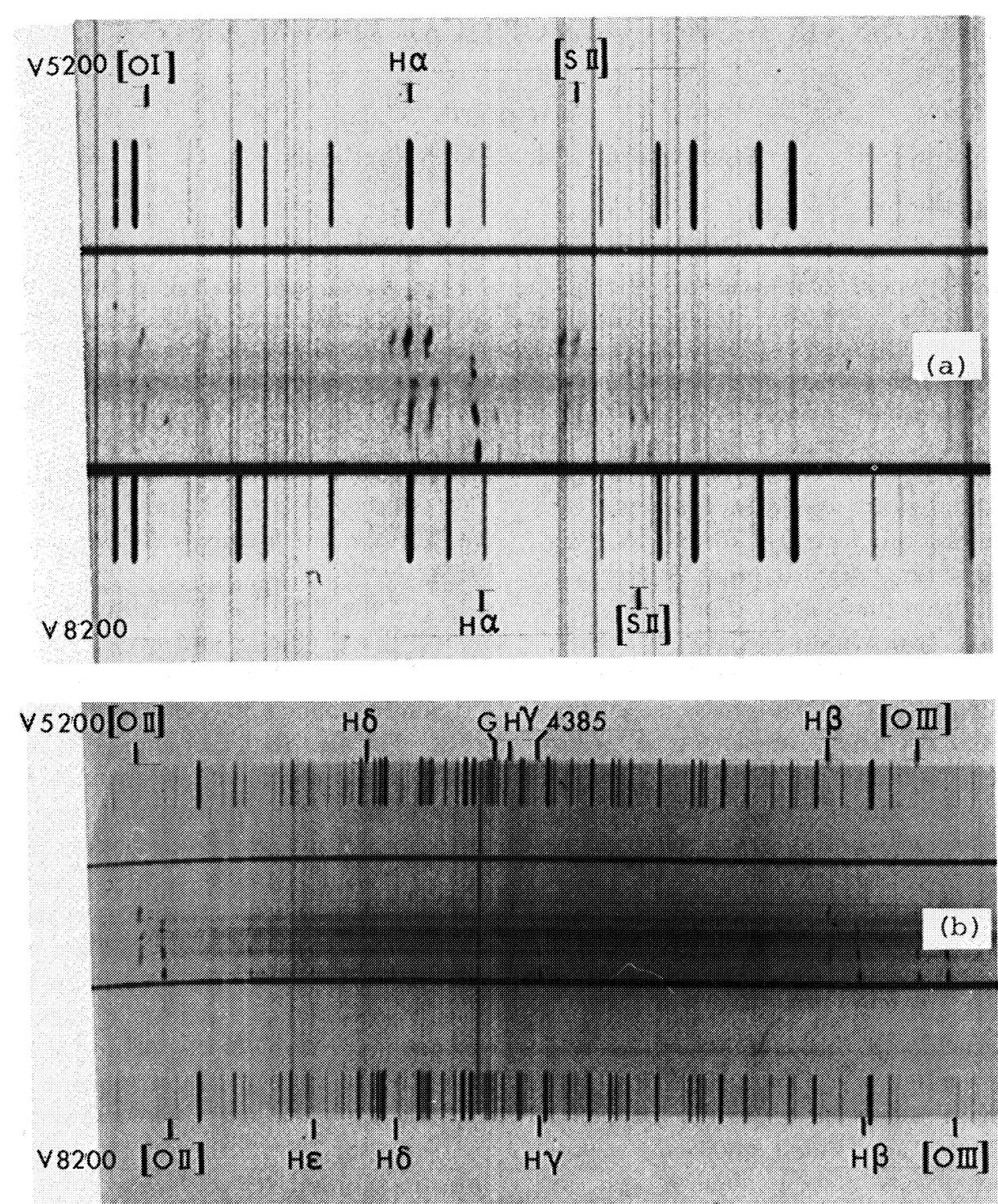

FIG. 1-Spectrograms with slit aligned roughly east-west and passing about $12^{\prime \prime}$ north of the nucleus of the $\mathrm{E}$ galaxy; dispersion $50 \AA \mathrm{mm}^{-1}$; (a) the red spectral region showing emission lines of $\mathrm{H} \alpha$ and [ $\left.\mathrm{S}_{\mathrm{II}}\right]$ in both systems, and [ $\mathrm{O}_{\mathrm{I}}$ ] in the high-velocity system; (b) the yellow-blue spectral region showing [ $\mathrm{O}_{\mathrm{III}}$ ], Balmer emission lines, [ $\mathrm{O}_{\mathrm{II}}$ ], and $\mathrm{H}$ and $\mathrm{K}$ in night sky and in galaxy, with $V=5200 \mathrm{~km} \mathrm{~s}^{-1}$; the G-band and $\lambda 4385$ absorption are also seen at this velocity. Note steep Balmer decrement and weak [O III] $\lambda 5007$ in the $V=5200 \mathrm{~km} \mathrm{sec}^{-1}$ system. Continuum arises from $5200 \mathrm{~km} \mathrm{sec}^{-1}$ stellar population; striping is due to obscuration from intervening $V=8200 \mathrm{~km} \mathrm{sec}^{-1}$ galaxy. Note that $8200 \mathrm{~km} \mathrm{sec}^{-1}$ emission is often strong where background continuum is obscured.

strikingly on the SE side of the center, where they extend to at least $7^{\prime \prime}(3 \mathrm{kpc})$. Toward the NW they are very much fainter. Again, this asymmetry is presumably due to absorption by the $8200 \mathrm{~km} \mathrm{~s}^{-1}$ system whose $\lambda 3727$ emission line is seen on the NW side, extending almost to the nucleus, but certainly not beyond.

The abundant A-type population may well be due, as van den Bergh (1972) has suggested, to a burst of star formation at the time of the expulsion of the $5200 \mathrm{~km} \mathrm{~s}^{-1}$ filaments.

Independent evidence indicating that the $8200 \mathrm{~km}$ $\mathrm{s}^{-1}$ system lies in front of the $\mathrm{E}$ galaxy has come from the discovery by DeYoung, Roberts, and Saslaw (1973), of a very narrow and deep 21-cm absorption line at a velocity of $8120 \mathrm{~km} \mathrm{~s}^{-1}$. The absorption is confined to a region $<6^{\prime \prime} \mathrm{EW}$ and $<9^{\prime \prime} \mathrm{NS}$ around the nucleus (Ekers, van der Hulst, and Miley 1976). It appears likely that the line is due to absorption of the strong radiation of the main galaxy's nucleus by an ordinary interstellar cloud in an intervening galaxy. DeYoung et al. have also discussed the alternative model of an expulsion from the nucleus. Upon closer consideration this leads, however, to great difficulties. The narrowness of the line combined with its great depth shows that it would have had to be ejected from 


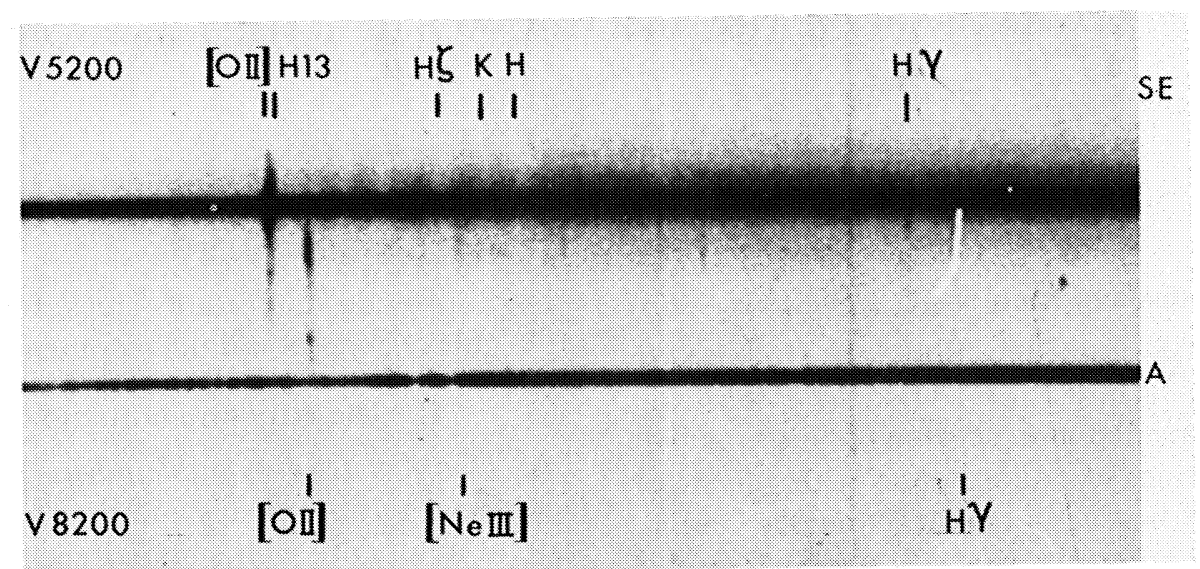

FIG. 2-Spectrum with slit in position angle $110^{\circ}$, passing through nucleus, showing early-type Balmer absorption spectrum southeast of nucleus, $V=5200 \mathrm{~km} \mathrm{sec}^{-1}$. Northwest of nucleus toward the bright star $\mathrm{A}$ at the bottom Balmer emission at both $V=5200$ and $V=8200 \mathrm{~km} \mathrm{~s}^{-1}$ is present, and the underlying continuum is weaker. The two bright emission lines are the [O $\mathrm{II}$ ] lines at $3727 \AA$ at 5200 and $8200 \mathrm{~km} \mathrm{~s}^{-1}$, respectively. The latter is seen to extend almost to the nucleus but is entirely absent southeast of it. Dispersion $26 \AA \mathrm{mm}^{-1}$. The distance between the nucleus and the star is $45^{\prime \prime}$.

one of the nuclear components in an extremely narrow cone and would be seen in absorption against another nuclear component behind it and situated within the same cone. Though this is perhaps not impossible the interpretation as absorption in an intervening galaxy seems at present more plausible.

The new spectroscopic observations were started with the aim of searching for absorption lines at 8200 $\mathrm{km} \mathrm{s}^{-1}$ which would confirm that the emission was connected with an independent galaxy rather than with ejected gas. This search has been unsuccessful. Although the $\mathrm{H}$ and $\mathrm{K}$ absorption lines, the $\mathrm{G}$ band, and two metallic line blends are clearly visible at the velocity of $5200 \mathrm{~km} \mathrm{~s}^{-1}$ there is no indication of any of these at $8200 \mathrm{~km} \mathrm{~s}^{-1}$. After all, this is not surprising, because it is clear from the abundance of emission knots that if the $8200 \mathrm{~km} \mathrm{~s}^{-1}$ filaments belong to a galaxy, it must be one of very late type. In such a galaxy the stellar surface brightness must be considerably lower than in the relatively dense part of the $\mathrm{E}$ galaxy in which the $5200 \mathrm{~km} \mathrm{~s}^{-1}$ absorption lines are observable. The absence of observable absorption lines in the $8200 \mathrm{~km} \mathrm{~s}^{-1}$ system does not therefore affect our general conclusion that the 8200 $\mathrm{km} \mathrm{s}^{-1}$ filaments belong to an independent late-type galaxy projected by chance on to one of the most explosive elliptical galaxies known. It may be noted that a similar conclusion has recently been put forward by Shields and Oke (1975) on partly the same grounds.

The $8200 \mathrm{~km} \mathrm{~s}^{-1}$ system gives evidence of a rotation with a speed such as would be expected for a normal medium-size galaxy. Combination of the new velocities with those measured by the Burbidges shows a systematic change from east to west (i.e., along the major axis) corresponding with a maximum rotation of about $150 \mathrm{~km} \mathrm{~s}^{-1}$.

NGC 1275 is situated in the densest part of the Perseus cluster (radius $\sim 400 \mathrm{kpc}$ ). The chance of finding a second galaxy within $30^{\prime \prime}$ of the nucleus of NGC 1275 is not prohibitively low, nor is the velocity difference unreasonably high; of the 13 known velocities in this part of the cluster three lie above $8000 \mathrm{~km} \mathrm{~s}^{-1}$. It appears unlikely that the two galaxies are in collision, as initially suggested by Mirkowski. A collision at such a high velocity would probably reveal itself by high-temperature radiation, while one would then also expect to find filaments of intermediate velocity.

The principal objection that may be felt against the interpretation of the $8200 \mathrm{~km} \mathrm{~s}^{-1}$ filaments as part of a galaxy is its somewhat unusual morphology. The numerous strong emission regions, and in particular the absence of a nucleus, correspond to a spiral of very late, possibly Magellanic type. Such a type is rare in dense clusters. We recognize the difficulty but consider that it is overruled by the practically convincing evidence that we are indeed observing a superimposed galaxy.

\section{REFERENCES}

Bergh, S. van den 1972, J.R.A.S. Canada 66, 237.

Burbidge, E. M., and Burbidge, G. R. 1965, Ap. J. 142, 1351.

DeYoung, D. S., Roberts, M. S., and Saslaw, W. C. 1973, Ap. J. $185,809$.

Ekers, R. D., van der Hulst, J. M., and Miley, G. K. 1976, Nature 262, 369.

Lynds, C. R. 1970, Ap. J. (Letters) 159, L151.

Minkowski, R. 1955, Carnegie Inst. of Washington Yearbook $54,25$.

— 1957, in Radio Astronomy, I.A.U. Symposium No. 4, H. C. van de Hulst, ed. (Cambridge: Cambridge University Press), p. 107.

— 1968, A.J. 73, 842 (Plate S-III).

Shields, G. A., and Oke, J. B. 1975, Pub. A.S.P. 87, 879. 